\section{CONTRIBUIÇÃO AO ESTUDO DO SISTEMA EXCRETOR DO FIGADO EM ANIMAIS SILVESTRES. V. VIAS BILÍFERAS DA CAPIVARA (HYDROCHOERUS HYDROCHOERIS)}

MARIA ANGELICA MIGLIND Professor. Assistente Doutora Faculdade de Medicina Veterinaria Zootecnia da USP

IRUENIA LUIZA DE SANTIS PRADA Professora Adjunta
Faculdade de Medicina Veteringria e Zootecnia da USP

WILSON MACHADO DE SDUZA Professor Assistente Doutor uldade de Ciencias Agrarias Veterinarias "Campus" Jaboticabal - UNESP

ANTONIO ALBERTO D'ERRICD

Professor Livre-Docente Faculdade de Medicina Veterinaria e Zootecnia da USP

AIGLINO, M.A.; PRRAR, I.L.S.; SOUZA, H.M.; g. ERRICD, H.A. Contribulejo go estudo do sisteme excentor do $f l g a d o$ an aniamis silvestres. $u$. vias billferas da cadivara (Hyarochoerus hyareenoeris). Rev.Fac.Med.vet.Zootec. Univ.j. Pзuเם, 23(2):115.132 \986.

RESUMO: Ef atuou-se a sistematizacyo das vias jiblferas de 15 eadivaras (Hydrochoerus hydraenoeris), adultos, sendo 10 nachos. 5 femeas, reaijnea injecyo com Neodrene batex 650', fixa-

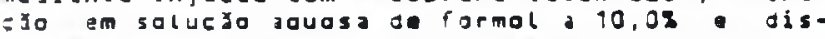
secesa. Ds esultados obtiaos aostrat qua: 13 o uluctus eholedocus resulta da tricanvergencia do - amus principalis dexeor. ramus principalis siiisier ducius systicus $(66,78)$, : amoem da uniro gireeg dos dois primeiros $(19,3 x)$ ou do ductus 7eoaticus duetus eysticus $(13,38)$; 23 o ductus neoaticus, caracterizado oela convergencza do pamus princiaalis dexter pamus grincipalis sinistar, duanoo, um delas nao se associa ductus eysticus, surge em poucos casos $(13,38)$. semorg livre da tpibutarios; 3) a Juetus eysti. eus livre de afluences $(92,4 x)$ ou a receoer tpon=a so ramus nedialis lool aextei pamus laoi Juadrati $(5,25)$, =oncore directamenta ara a jormaço do Juctus choladocus, unindo-se por : : Eanvergencia 30 imus princioalis dextar. - jmus princigalis sinister (66.75) ou do ductus reoaticus (13,25) incoraoranco-se, no pestante tas jecus $(19,38)$, ao sistema do pumus principalis daxter; t) o sistema do ramus principalis jexiar zcna-se integraao, de nodo geral, jelo amus lodi dextri bateralis $(100,08)$, pamus arocessi caudati (900,0s), pamus loaj dextri Tedialis $(92,+8)$, Jucius cysticus $(19,38)$ e pamus ooi guadati $(6, j 8)$ alem ge variavel numero ae zaneribuiedes inominaas do laous dexter laterabs. loous dexter neaialis, loous caudatus cars suoradortalis. processus aaoillaris a procassus : zuatus) : (aqus auadatusi j) a sistema do ramus princioalis sintster mostra-se constituldo, da nodo geral, pelo ramus labi sinistri lateralis $(100,05)$, pamus lobi sinistri nedialis $(900,08)$. pamus bobi quadrati ( 85.85 ), afora contribuicoles inominadas do lobus sinister lateralis, lobus sinister medialis, lobus quadratus lobus eaudatus (pars suoraportalis orocessus paoillaris).

UNRTERMOS: 1. Anatonia, Suldaet 2. Ductos biliares: 3 . Figadoit. Capivaras.

\section{INTRODUCAD E LITERATURA}

A sistematizasao dos companentes do sistema excretor do figado dos animais domasticos e silvestres constitui uma das linhas de pesquisa em desenvolvimento dentro das Disejplinas de Anatomia da Faculdade de Medicina Veterinaria a Zotecnia da U.S.P., com o objetivo nJo apenas de obter dados de carater comparativo mas tambem, visando completar os dados genericos contidos nos tratados de Anatomia Veterinaria.

Considerando particularmente os poedores le aqui tamoem os coelhos, emoora sejam as vezes referidos a parte, eamo (agomorfos), encontramos poucos tradalinos que, como o prasente, efetuam sistematizac\%o dos coletores glandulares, ou seja, a de otTAUIANI, 5 (1933), em diversas especias - coetho, marmota, nutria e cobaia, o de DIAS et alii, 1 (1979), en coelhos, o de OLIVEIRA et alii, 4 (1979) en codaia e de PRPDA et atil, G(1981), em patzo do bannado.

otTAUIANI, 5 (1933), utibizando algumas especies como o coelho, a marmota, a nutria e a cobaia a valenda-se de radiografias, relata que nao existem na soelha, ductos que permitam distingubr dois terpltorios billferos, un direito un esauerdo, mas aponta a existencia de um so oucso billfero que nasce por neio de dois afluenles primbrios, aos quais enegam outros afluentes orimarios volumosos a dueto clstico. o colédoco e a continuacáa deste anico ducto bilifero e recolhe, na sua ultima parte, o afluente primario do laoo caudato. Para a narmota, dizo autor que năo se pode distinguir um dueta laoar direito de um esquerao, senao as territarias direita esquerdo mal geíinidas. Is dais ductos, nascem por neia de varios afluentes primarios que, uninao-se am " $V$., constituem - ducto bibliero intra-nepatico. Os afluentes do lobo eaudato, por sua vez, disademse radialmenta e se unem oara constituir um consolcuo af luente primbrio que penetra no coledoco. Algumas vezes enconita-sa um ducto direito a um asquerdo ou im só aucto, canstituido por um jibuente primaria do ducto direito de varios afluentes orimarios do looo esquerdo. Neste unico ducto pode aesemoocir a ducto do boo caudaco. Pelativamente a nutria, descreve o autor 
Que o complexo das vias biltferas intra e extra-hepaticas assume disposica em "T". Nos territorios de afluxo, os dois ductos กรо sรo sempre os mesmos, mas geralmente uma linha que passa pela fossa cistica e ponto de encontro dos dois ductos biliferas intra-hepaticos, divide o flgado em dois territorios, direito e esquerdo, cada um dos quais é provido de ducto proprio. Excepcionalmente, o lobo medial direito es tributario do ducto esquerdo. Na parte direita a lobo caudato, existe grande quantidade de afluentes biliferos que se unem em un unico que por sua vez participa da constituicro do ducto direito. Quase sempre - lobo esquerdo possui tres a quatro afluentes primbrios que constituem o ducto billfero de modo diverso de 5 aso para caso. Para esse autor, na cavia adulta, o flgado e dividido nos territorios direito e esquerdo, cada um dos quais é percorido por um ducto bilifero intra-heoatico. Do ducto direito săo tributários, um afluente primário do lobo mediano direito e um ou dois afluentes primbrios do lobo caudato. En alguns individuos, o alfuente primbrio do lobo medial direito comeca por meio de muitos afluentes secundarios, distribuldos em leque. Ele desemboca na parte ventral da ultima porço do ducto bilifera direito, mas em alguns casos pode desembocar na grande dilataço formada pelo encontro do ducto direito com o ducto esquerdo. Excepcionalmente, o lobo medial esquerdo fornece un afluente primario que correndo no parenouima, cruza eranialmente o ducto clstico e termina no ducto billfero direito. Pode-se observar, enfim, que o lobo medial direito, junto ao lobo medial esquerdo, possui um so afluente primbrio que desemooca ora no ducto direito, ora no ducto esquerdo ou no ponto de encontro dos dois ductos. Quanto ao lobo quadrado, a sua porço aderente ao lobo direito e drenada por un afluente orimario que desemboca no ducto billfero direito e se junta em " $V$. com o unico af tuente primario que atravessa a parte relacionada a veia cava. Quando se encontra esta disposicro, o ducto resultante e situado sobre uma linha sagital que passa pelo ponto de encontro dos dois ductos looares e muito freqóntemente, este tronco desemboca na dilatacgo formada no ponto de uniro dos dois ductos, podendo ainda desembocar no ducto direito ou no esquerdo. O ducto esquerdo e mais longo e fino que o direito e nao se observam anastomoses entre os varios afluentes secundarios que se unem em leque para constituir - dueto billfero esquerdo. Talvez entre looo medial esquerdo e o direito exista um pequeno lobo (lobo quadrado), provido de um fino afluente primario que desemboca no ducto billifero esquerdo.

Jifs et alii, 9 (1979), utilizando 34 flgados de coelhos adultos e valendo-se de estudo anatomo-radiografico, concluem que o ducto coledoco e formado por apenas duas ralzes, ramo principal direito e ramo principal esquerdo e recebe contribuifoes do processo caudado, de todo o lobo caudado e processo papilar; o ducto elstico integrase ao sistema do ramo principal direito $(94,118)$ ou ao sistema do ramo principal esquerdo $(5,8 \%)$; participam do sistema do ramo principal direito, mais freqoentemente $(76,47 \%)$, os ramos lateral e medial do lobo direito e o ducto clstico e, ainda, o pamo do processo papilar, ramo do processo caudal, ramo do lobo quadrado e ramo do lobo intermedio; integram o sistema do ramo principal esquerdo, com maior frequencia (52.94\%), e ainda, ramos do lobo quadrado, do tovo intermedio e do lobo esquerdo, alnda, do processo papilar, do labo direito, do lobo quadrado e o ducto clstico.

OLIVEIRA et alid, 4(1979), efetuando a sistematizacăo do ramus principalts sinister de 44 cobaias adultas mediante corroşo, dissecço e radiografias, relatam, segundo pudemos apurar, que a esse sistema incorporam-se habitualmente o ramus lateralis lobi sinistri e o ramus medialis lobi sinfstri, aos quais podem se juntar ainda o ramus lobi quadrati $(59,0 \%)$; este e o ramus processi papillaris $(16,0 \%)$ e os tres primeiros mais ramus principalis dexter $(2,3 \%)$. Nas dois esquemas apresentados no trabalho, correspondentes a drenagem bilifera do flgado todo, muito embora estejam referendados adenas os coletores comuonentes do sistema do ramus principalis sinister, pudemos perceber que, no atinente ao primeiro, o ramis principalis sinister, antes de unir-se ao ramus principalis dexter para compor o ductus choledocus, recebe o ductus cyticus. Parece-nos que, no relativo ao segundo esquema, surge disposifăo contrária, isto e, o ductus cysticus incorpora-se ao sistema do ramus principalis dexter para depois ligar-se ao ramus principalis sinister, formando assim o ductus choledocus.

PRADA et alii, 5 (1981) estudaram o sistema excretor do flgado de 15 ratzes do banhado (Mlocastor coypus), 14 machos e 1 femea, adultas e concluliam cue a ductus choledocus, livre de tributarios em todas as pecas, mostra-se constituldo pela uni zo do ramus principalis dexter e ramus principalis sinister - 9 vezes $(60,0 \%)$, pela triconvergencia destes dois ramos e ductus cysticus - 3 vezes $(20,0 \%)$, bem como pela confluancia do ductus hepaticus mais ductus cystleus - 3 vezes $(20,0 \%)$; o ductus hepaticus identificado em 3 casos $(20,0 \%)$, exibindo-se livre de af buentes, resultando sempre da assocjacáo do ramus principalis dexter e ramus principalis sinister; o ductus cysticus integra o sistema do ramus principalis sinister - 5 vezes $(33,3 \%)$, o sistema do ramus princioalis dexter - 4 vezes $(26,7 \%)$ e associa-se aos dois citados ramos, por triconvergencia - 3 vezes $(20,0 \%)$ e ao ductus hepaticus - 3 vezes $(20,08)$; integram o sistema do ramus prin- 
cipalls dexter, o ramus processi caudati, o ramus loai dextri lateralis, o ramus lobi dextri medialis e por vezes o ductus eysticus, alem de numerasas contribuicales procedentes do lobus dexter laterabis, lobus dexter mediabis e loous caudatus lpars supraportalis, processus papilaris e processus caudatus); participan do sistema do ramus prineipalis sinister, o ramus lobi sinistri lateralis, o ramus lobi sinistri medialis, o ramus lobi quadrati e, por vezes, a ductus cysticus, afora numerosas contribuifbes vindas do lobus sinister lateralis, lobus sinister nedialis, lobus quadratus, lobus caudatus (pars supraportalis e processus papi(larís) e lobus dexter nedialis.

\section{MATERIAL E METODO}

As preoaracoses componentes deste trabalho foram obtidas de 15 cadivaras (Hydroehoerus hyorochoeris), adultas, 10 maenos e 5 femeas, provenientes dos munieiojos de Rio Verde, Coxim e Aquidauana (MS - Pantanal Matograssense). Cada peca era consti. tulda apos reducyo, do figado. tracto duodenal correspondente d desembocadura do coledoco. Depois de conveniente esvazlamento, o sistema billfero era injetado, atraves de canatizacyo do ducto coledoco pela papila duodenal maior, eom Neoprene latex 650', corado com pigmento especifico. $A$ seguir, os brgyos eram fixados en solueyo aquosa de formol a 10,08 , dissecados, esquenatizados alguns fotografados para posterior documentacso.

Como propoem NICKEL, et alii, 3, pelativamente aos animais domesticos de conformidade com o procedimento, nessa sentido, da linha de pesquisa 3 que nos referimos, encontramos candicáes, em nosso matepial, de igualmente considerar no flgado da capivara, tres partes, determinadas por dois planos paralelos entre si e perpendieulares as superfleies diafragmatica e visceral do orglo, tracados em correspondencia a alguns acidentes anatomicos. Deste modo, um deles sobrepore-se ao maior eixo da fossa da vesicula biliar atinge dorsalmente a impressyo da veia cava caudal, enquanto o outro aleanca a incisura do ligamento redondo a Impressyo esof agica. Definimos, assim, as porebes direta, intermedia esquerda da glandula, nas quais identifleamas, obedecendo orientacto dos tratadistas mencionados, e valendo-nos das particularidades anatonicas que o orgyo apresenta, por orden, o lobus dexter late$r a l i s e$ o lobus dexter nedialis o boous sinister lateralis e o lobus sinister medialis, o lobus quadratus, na porero Infra-portal, convencionalmente separado do lobus dexter medialis por um dos planos tracadosi o lobus eaudatus, integrado pelo processus papillaris, e pars supraporalise processus caudatus que se expande a direi- ta sooreoondo-se a parte do labus dexter lateralis.

Adotamos, no relato dos resultados, a mesma designacyo das principais vias coletoras dos looos, prodosta dor JABLAN-PANTIC, 2 (1953), em : iavalhos relativos a figados lobados de alguns animais (sulno, eyo e gato\}. Por outro lado, apenas consideramos, na deseriço dos resultados, as ralzes das vias coletoras dos looos, somente quando ligada a af luentes oriundos de outras regibes glandulares. $N a$ designacao dos contingentes quabificados por nomes proprios, mais do que seu caliore, levamos en consideracto as dimenzoes da correspondente zona de escoamento, visto que as injectes podem facilitar o preenchimento das vias superficiais.

Por altimo, esclarecemos, na sistematizacso das vias biliferas da capivara, a citacyo dos coletores obedeceu ao sentido periferia - centro de sua disposiczo.

\section{RESULTADOS}

Na sistematizacá das vias biliferas Intra extra-heoaticas da eapivara CHydrochoerus hydrochoeris), obtivemos os seguintes resultados (Fig. 1 a 3 ), que estgo expostos de acordo com os seguintes itens:

A) DUCTUS CHOLEDDCUS

B) DUETUS HEPATIEUS

(C) DUCTUS CYSTICUS

D) SISTEMP DO RAMUS PRINCIPRLIS DEXTER

E) SISTEMA DO RAMUS PRINCIPRLIS SINISTER

F) TRACTOS ANASTOMOTICDS

\section{ค) DUCTUS CHOLEDDCUS}

Nos 15 flgados analisados, verifleamos - ductus choledocus (Figs. 1 a 3 ), senore live de tributarios, a resultar da triconvergencia do ductus cysticus, pamus prineipalis dexter a ranus principalis sinister, 10 vezes 666,78 - Obs. $1 \mathrm{~m}, 2 \mathrm{f}, 3 \mathrm{f}, 4 \mathrm{f}, 8 \mathrm{f}$, $10 \mathrm{~m}, 11 \mathrm{~m}, 12 \mathrm{~m}, 14 \mathrm{~m}, 15 \mathrm{f})$, da uniyo dipeta do ramus principalis dexter con pamus princlpalis sinister. estando o ductus eysticus incorporado a sistema do primeiro deles - 3 vezes $(20,0 \%$ - Obs. $6 m, 7 m, 9 m)$, - ainda a originar-se da reunigo do duetus cysticus com o ductus hepaticus, 2 vezes $(13,38$ - Dbs. $5 n, 13 m)$.

\section{B) DUCTUS HEPRTICUS}

Dentre as 15 pecas dissecadas, vimos o ductus hepaticus, caracterizado a partir da unigo do ramus principalis dexter com pamus principalis sinister, 2 vezes $(13,38$ - Obs. $5 m, 13 m$, mostrando-se em amoas as pecas, livre de af buentes.

\section{(c) DUCTUS EYSTICUS}

0 ductus eysticus surge livre de tributarios en 14 preparacaes $(92,4 \%$ - Obs. 
1m, 2f, 3f, 4f, $5 \mathrm{~m}, 6 \mathrm{~m}, 7 \mathrm{~m}, 8 \mathrm{f}, 8 \mathrm{~m}, 10 \mathrm{~m}$, $11 \mathrm{~m}, 12 \mathrm{~m}, 14 \mathrm{~m}, 15 \mathrm{f})$, recebendo no caso comolementar $(5,78$ - Obs. $13 \mathrm{~m})$, tronco comun formaco pelo ramus medialis lobi oextri e ramus looj quaorati, troneo ao qual vem ter contribuigao oo lobus dexter medialis. Na maioria das pecas, vale dizer, 10 vezes $(66,7 \%$ - Obs. $1 \mathrm{~m}, 2 f, 3 f, 4 f, 8 f$, $10 \mathrm{~m}, 11 \mathrm{~m}, 12 \mathrm{~m}, 14 \mathrm{~m}, 15 \mathrm{f})$, o ductus eysticus une-se ao pamus principalis dexter $e$ ao ramus principalis sinister, caracterizanda uma triconvergeneia para constituiero do ductus choledocus. Em menor número de casos, isto e. 3 vezes $(20,03$ - abs. $6 \mathrm{~m}, 7 \mathrm{~m}$, 9m), o ductus cysticus incorpora-se do sistema do ramus principalis dexter. Eectarecemos, ainda, que em apenas duas oporrunjdades $(13,3 \%$ - Obs. $5 \mathrm{~m}, 13 \mathrm{~m})$, o ductus cysticus une-se ao ductus hepaticus representando, o tronco formado, o proprio duetus choledocus.

\section{SISTEMA DO RAMUS PRINEIPRLIS DEXTER}

Nas 15 preparacbes estudadas, forman o sistema do pamus principalis dexter, o ramus lobi dextri lateralis, o pamus processl caudati o ramus lobi dextri medialis, 14 vezes ( $92,4 \%$ - Dbs. Im, $2 f, 3 f$, $4 f, 5 m, 6 m, 7 m$, of $9 f, 10 m, 11 m, 12 m, 14 m$, 15m). Integra, tamben, o aludido sistema, alem desses ductos nominados, o ductus cysticus, 3 vezes $(20,08$ - Obs. $6 \mathrm{~m}, 7 \mathrm{~m}$, $9 \mathrm{~m})$ e o ramus lobi quadrati, 1 vez $(6,78$ Obs. 10m). D ramus principalis dexter resulta apenas da confluencia do ramus lobi dexter lateralis e do ramus processi caudati, 1 vez (5,7s - Obs. 13m). Nas 15 peeas, a lem desses coletores indicados, integram o referido sistema, diversos afluentes inominados vindos do lobus dexter lateralis, lobus dexter medialis, lobus caudatus iprocessus caudatus, processus papillaris e pars supraportalis) e lobus quadratus.

Vejamos, agora, as diversas associaEbes que estabelecem, entre si, os coletores nominados integrantes do sistema do ramus principalis dexter, salientando o seu comportamento bem como o da via que formam.

1) 0 pamus lobi dextri lateralis o ramus processi caudati contribuem para a formacso de ducto comum em 13 das 15 preparactes $(85,85$ - Obs. 1m. 2f, 4f, 5m, 7m, 8f, 9m, 10m, $11 m, 12 m, 13 m, 14 m, 15 f)$. Ambos surgem livres de contribuicbes en 8 pecas (52,8s - Obs. $1 \mathrm{~m}, 2 \mathrm{f}, 8 \mathrm{f}, 9 \mathrm{~m}, 10 \mathrm{~m}$, $11 \mathrm{~m}, 14 \mathrm{~m}, 15 \mathrm{f})$. Daqueles 13 orgaos (85, 85 - Obs. im, $2 f, 4 f, 5 m, 7 m$, Bf, $9 m, 10 m, 19 m, 12 m, 13 m, 14 m$, 15f), o ramus lobi dextri laterabis recebe coletores inominados em 2 deles (13,3\% - Obs. 4f, $7 \mathrm{~m})$, ou seja, apenas un afluente inominado procedente do lobus caudatus (processus caudatus), 1 vez ( $8,7 \%$ - Dbs. 4f) e un coletor consplicuo dessa mesma ari- gem, 1 vez $(6,7 \%$ - Obs. $7 m)$. 0 pamus processi caudati surge livre de afluentes nesta 2 glandulas. Este coletop nominado, dentre aquelas ia Decas (85,8:- Obs. $1 \mathrm{~m}, 2 f, 4 f, 5 \pi$, $7 \mathrm{~m}, 8 \mathrm{f}, 5 \mathrm{~m}, 10 \mathrm{~m}, 11 \mathrm{~m}, 12 \mathrm{~m}, 13 \mathrm{~m}$, $14 \mathrm{~m}, 15 \mathrm{f})$, acolhe tributarios en 3 delas $(19,8 \%$ - Obs. $5 m, 12 m, 13 m)$. oriundos:

a) somente do lobus dexter lateralis, ou seja, dois eferentes em tronco, 1 vez $(6,78$ - Dbs. 5m), dois afluentes isolados dessa mesma origem, o primeiro deles consplcuo, 1 vez (E,78. Obs. $12 \pi)$ :

b) simultaneanente do lobus dexter lateralis e do lobus caudatus (processus caudatus e pars supraporta(ls), isto e tres afluentes, o primeiro deles isoLado, procedente do lobus dexter lateralis e os dois ultimos formando tronco comum, oriundos do loous caudatus (processus caudatus e pars supraporta(is), 1 vez ( $6,7:$ - Obs. $13 \mathrm{~m})$. A via para a qual confluem o ramus lobi dextri lateralis e o panus processi caudati surge livre de coletores em unica oportunidade $(6,74$ - Obs. 10m); nas demais dissectores $(79,2 \%$ - Obs. 1m, 2f, 4f, 5m, 7m, 9m, 11m, 12m, 13m, $14 \mathrm{~m}, 15 \mathrm{f}$ ) acolthe af luentes de diversas origens, ou seja:

a) concomitantemente do lobus caudatus (processus papillaris e pars supraportalis) e do lobus dexter medialis, 3 vezes $(19,88$ - Obs. $1 \mathrm{~m}, 11 \mathrm{~m}, 15 \mathrm{f})$, ou seja, tronco camum de dois elementos, procedentes do lobus dexter medialis, seguido de afluente isolado oriundo do labus caudatus que reune duas contibuicoes do processus papillaris e una da pars supraportalis, 1 vez $(6,7 \%$. Obs. Im); tronco comum associando coletor consoicuo do lobus caudatus (pars supraportalis e processus papil(aris) $e$ afluentes do lobus dexter medialis, 1 vez $(6,7 \%$ - Obs. $11 \mathrm{~m})$ e tronco comum a tres coletores do lobus caudatus (um do processus papillaris e dois da pars supraportalis), seguido de dois aferentes da pars supraportalis e de outro tronco comuma tres af luentes do lobus dexter medialis, o segundo destes, conspicuo, I vez $(5,75$ - Dbs. 15f);

b) somente do lobus caudatus (processus papillaris e pars supradortalis), 3 vezes $(19,8 \%$ - Obs. $4 f, 13 m, 14 m)$, is to e dois coletores consoicuos da pars supraportalis, reunidos em tronco, ? vez ( 5.78 - obs. 4f), coletor isolado conspicuo que, redne una contribuicso de cada regilo, 1 vez (E, 7\% - Dbs. 13m) e tres coletores isolados 
da pars $(6,78-0 b s .14 m)$;

simultaneamente do lobus eaudatus (pars sudragortalis e processus paojl(arjs), lobus aexter laterabis e boous dexter medialls, 2 vezes $(13,3 \%$ - Obs. $2 f, 7 m), v a l e d i z e r$, afluente isolado do lobus dexter lateralis, seguido de autro, consojeuo, do lobus caudatus (processus paplllaris e pars supraportalis) e de tronco comuna quatro elementos do lobus dexter medialis, reunidos dois a dois, 1 vez $65,75$ - obs. $2 f)$ eferente isolado oriundo do lobus dexter lateralis. coletor do lobus caudatus (pars supraportalis), tronco comum reunindo tres coletores daquela mesma origen, tributarios consoleuo do lobus caudatus (reunindo contribuicso do processus papillaris e da pars supraportalis) e finalmente, afluente isatado do lobus dexter medialis, 1 vez $(6,7:$ - obs. $7 \mathrm{~m}$;

d) apenas do lobus dexter medialis, ou seja, dois tributarios isolados, 1 vez $(5,7 \%$ - Qbs. 5m);

e) concomitantemente do lobus eaudatus (pars supraportalis) do lobus dexter lateralis, vale dizer, afluente isolado do segundo labo, sucedido por tronco comum a dois componentes oriundos do lobus caudatus loars supraporta(is), un dos quais conspleuo, 1 vez $(8,7 \%$ - Dbs. 8f $)$;

f) somente do lobus dexter lateralis, isto e, dois coletores isolados, 1 vez $(6,78-0 b s .9 m)$.

g) slmultaneamenta do lobus dexter lateralis, lobus caudatus (processus caudatus pars supraportalis) e lobus dexter medialis, ou seja, tronco comum - coletor inominado procedente do lobus dexter lateralis a a dois af luentes do lobus caudatus (processus caudatus e pars supraportalis), seguido de dois tributbrios isolados, o primeiro oriunda do lobus dexter medialis e o segundo do lobus eaudatus (pars supraportalis), 1 vez ( 6,78 . obs. 12a).

2) 0 ranus lobi dextri lateralis e o ramus lobi dextri medialis constituen tronco conum, en apenas 1 preparacyo $(6,78$ - Obs. Gm). O primejro destes contingentes $b$ livre de cantribuifoses e a segunda recede afluentes vinaos, simultaneamente, do lobus quadratus, dois afluentes isoladose, da labus dexter lateralis, un eferente. O tronco conum que formam e tamben isento de contribuicoses.
3) 0 ramus processi caudati e o ramus lobi dextri medialis comporem via comum, en 1 caso $(5,7 \%$ - abs. $3 f)$, oportunidade em que ambos recebem contribuicores inominacas de diferentes origens. Deste modo, o ramus processi caudati acolhe dais afluentes, o primeiro do lobus dexter medialis e o segundo, conspleuo, do lobus caudztus (pars supraportalis). 0 ramus lobi dextri medialis, a seu turno, acolhe dois afluentes do lobus quadratus, o primeiro isolado e a segundo comoondo tronco com contingente inominado do lobus caudatus (oars supraportalis).

4) 0 ramus lobi quadrati e o ramus lobi dextri medialis confluem para tronco, en 1 peca (5,75 - Obs. 10m), surgindo l lure de tributbrios enquanto ao ejtado tronco vem ter tres eferentes do lobus dexter medialis, o drimejro isolado e os outros dois em tronco com tributarios vinde da pars sudraortalis e coletor do processus paoiblaris, sucedendo-os via originaria do loous quadratus.

Observemos agora a maneira pela qual, continuando a confluir, os tributbrias nominados integrantes do sistema, do panus principalis dexter, determinan a sua formacar.

1) 0 ramus lobi dextrl lateralis contribuj para a formacya do ramus principalis dexter, ea todas as preparasos, quando o encontramos associados ao ramus processi caudat 1,13 vezes (85,85 - Obs. 1n, 2f, $4 f, 5 n, 7 n, 8 f$, 8m, 10m, 11m, 12m, 13m, 14m, 15f). Nas outras 2 pecas, observamos este coletor nominado unindo-se em tronco comum com a ranus processi caudati mais ramus nedialis lobi dextri, 1 vez $(5,78$ - Obs. 3f). A via comun, aqui constitulda, pepresenta o proprio ranus principalis dexter encontra-se isento de contribuicoles. Na outra glandula $(6,58$ - Obs. $5 m)$, comoonente deste grupo, vimos a coletor em foco, compondo tronco comum com o ramus lobl dextri nedialis, oportunidaoe em aue se encontra livre de afluentes.

2) 0 panus processi caudat1, conforme verificamos, encontra-se diretamente unido ao ranus lobi dextri lateralis, 13 vezes $(85,85$ - Obs, $1 \mathrm{~m}, 2 f, 4 f, 5 f$, $7 \mathrm{~m}, 8 \mathrm{f}, 3 \mathrm{~m}, 10 \mathrm{~m}, 11 \mathrm{~m}, 12 \mathrm{~m}, 13 \mathrm{~m}, 14 \mathrm{~m}$, 15f). Nas duas peeas complementares $(13,28$ - Obs. $3 f$, on), vimos esta componente formando tronco camum can o ramus lobi dextri nedtalis, 1 vez65,74 - Obs. 3f), oportunidade en que amoos acolhem eferentes de origens diversas, vale dizer, ao ranus lobi dextri nedialis afluem tres componentes, a 
primeiro isolado, vindo do lobus quadratus $e$ o segundo, desta mesma origem, compondo tronco com eferente do loous caudatus coars supraoorta(i5). Quanto ao ramus processi caudati, nesta oreparacao, acolne oois afluentes, o primeiro do lobus dexter medialis e o ultimo, conspleuo, oo labus caudatus (pars supraportalis processus caudatus). O ducto comum aqui formado encontra-se isento de contribuictes. Na outra dissecego (6,7\% - Obs. 5m), assinalamoso ramus processi caudati uninoo-se ao tronco constituido pelo pamus lobl dextri lateralis e ramus labi dextri medialis, acolhendo, nesta preparacyo, dois coletores conspicuas do lobus caudatus (pars supraportalis), ao segundo deles unindo-se contribuicso do processus padillaris. A via comum assim constitulda encontra-se isenta de contribuisores.

3) D ramus lobi dextri medialis, segundo referimos, encontra-se unido so tronco constituldo pela confluencia do ramus processi caudati e do ramus lobi dextri laterolis, 11 vezes $(72,65$ Obs. 1m, 2f, $4 f, 5 m, 7 m, 8 f, 9 m, 11 m$, $12 \mathrm{~m}, 14 \mathrm{~m}, 15 \mathrm{f}$ ), 20 ramus lobl quadrati, 2 vezes $(13,35$ - Obs. $10 \mathrm{~m}, 13 \mathrm{~m})$, ao pamus processi caudati, 1 vez $65,7 \$$ - Dbs. Jf) e ao ramus lobi dextri lateralis, 1 vez $(6,78$ - Obs. $6 \mathrm{~m})$. No grupo de 19 pecas $(72,68$ - Dbs. 1m, $2 f, 4 f, 5 m, 7 m$, of , 9m, 49m, 12m, 14m, 14f), en que o ranus lobl dextrl medialis une-se ao tronco comun oriundo da uniso do ramus processi caudati e do ramus lobi dextri lateralis, ele acolhe tributarios de diversas origens, isto :

a) Somente do bobus quadratus, 7 vezes $(46,2 \%$ - Obs. $1 \mathrm{~m}, 4 \mathrm{f}, 5 \mathrm{~m}, 19 \mathrm{~m}, 12 \mathrm{~m}$, $14 \mathrm{~m}, 15 \mathrm{f})$, melhor explicando, dois coletores isolados, 4 vezes $(26,4 \%$. Obs. 1m, 4f, 5m, 11m), un tributario consplcuo, 9 vez $(5,75$ - Obs. $12 \mathrm{~m})$, ou กรo, 2 vezes $(13,28$ - Obs. $14 \mathrm{~m}, 15 f)$. o ducto caracterizado nestas glandulas =onstitui o proprio ramus principalis dexter e surge livre de eferentes, 5 vezes $(33,0 \%$ - Obs. 1m, 4f,12m,14m,15f) - acolhe nas demais preparacoses $(13,38$ - Obs. $5 \mathrm{~m}, 11 \mathrm{~m}$ ) af luentes orjundas concomitantemente do lobus quadrarue, lobus oesitar medialis e lobus caudatus (pars supraportalis e processus papil(aris), 1 vez ( $6,7 \%$ - Dbs. $5 \mathrm{~m}$ ), nelhor exolicitanao, auatro coletores, os tres primeiros isolados, dos quals o primeiro e o terceiro do lobus auadratus, o segundo do lobus dexter medialis o ditimo, conspleuo, do lobus caudatus, reunindo contribuicyo da pars supraportalis e do processus papillaris; na outra observasyo $(6,78$
Dos. $11 \mathrm{~m}$ ), o ramus principalis dexter recebe un tributario isolado 00 lobus caudatus (pars supraportalis);

b) simultaneamente do lobus caudatus (pars supraportalis) e do loous quadratus, 2 vezes (13,3\% - Obs. af, $9 m$ ) ou seja, tres coletores, o primeiro independente do lobus quadratus $e$ os dois ultimas, formando tronco conum, um de cada lobo, 1 vez $65,7 \%$ Obs. Br) e sete tributarios, os einco primeirosidsolados do. Lobus quadratus e os dois ultimos, comporm tronco comum, um do lobus quadratus a outro do lobus eaudatus (pars supraportalis e processus papillaris), 1 vez (5,75 Dbs. 9m). D dueto comum visto nestas duas preparacbes, constitui ja o proprio ranus principalis dexter e encontra-se livre de afluentes;

c) concomitantemente do lobus auadratus e do labus dexter medialis, i vez $(E, 7 \%$ - Obs. 2f), vale dizer quatro ailuentes reunidos em dais troncos de dois elementos, as primeiros originando-se do lobus quadratus e os ditimos, un do lobus dexter medialis a autro daquele lobo. A via comua, aqui caracterizada, representa o ramus prineipalis dexter - encontra-se livre de afluentes;

d) Simultaneamente do lobus quadratus, lobus dexter medialis e lobus caudatus (pars supraportalis), 1 vez (6,7\%Obs. $7 \mathrm{~m}$ ), isto tronco camum de dois componentes, un do lobus dexter medialis a outro do lobus caudatus cpars supraportalis), seguido de quatro componentes vindos do lobus quadratus, os tres peimeiros reunidos em troncos. D conducto verificado nesta peea pepre senta, ja, o proprio ramus principalis dexter a acha-se isento de contribuicotes.

\section{E) SISTEMA DO RAMUS PRINCIPQLIS SINISTER}

Nas 15 preparactes estudadas, observamos a fazerem parte do sistema do pamus principalis sinister, o ramus lobi sinistri lateralis $e$ o ramus labi sinistri medialis, ea todas alas, e o ramus lobi quadrati, en 13 destas pecas $(85,8 \%$ - Obs. $1 \mathrm{~m}, 2 \mathrm{f}, 3 \mathrm{f}$, 4f, 5m, 6m, 7m, of, 9m, 19m, 12m, 14m, 15f), afora contribuieśes inominadas vindas do lobus sinister lateralis, lobus sinister medialis, lobus quadratus e lobus eaudatus corocessus padiliaris e oars supraporta(1s).

Descreveremos, a seguir, as diversas associacbes diretas que estabelecem entre si, os coletores nominados integrantes do sistema do ramus principalis sinister, salientando o comportamento de cada un deles $e$ o da via comum resultante. 

capivara (Hydrochoerus hydrochoeris).

D ramus lobi sinistri medialis e pamus lobl quadrati comporm tronco comum en 9 das 15 preparacoes $(59,48)$, ambos encontrando-se livres de tributários, 6 vezes (39,7: - Obs. $1 \mathrm{~m}, 2$ ? , $7 \mathrm{~m}, 8 f, 12 \mathrm{~m}, 14 \mathrm{f})$. Tanto o ramus looi sinistri medialis como o ramur lobi quadrati acolhen tributarios de diversas origens, 1 vez (5,78 - Obs. $9 m$ ), vale dizer, ao primeiro deles afluem quatro tributarios isolados vindos do lobus quadratus, enquanto o segundo recebe eferente do lobus sinister medialis. Somente o ranus lobi quadra$t i$ acolhe contribuiczo inominada, 1 vez (s.7s - Obs. $11 \mathrm{~m})$, isto e, af luente isolado conspleuo do lobus sinister mediells. Finatmente, em 1 unica preparacso $(6,78$ - Obs. $14 \mathrm{~m})$, apenas 0 pamus lobi sinistri medialis acolhe eferentes, vale dizer, tronco comum de dois componentes do lobus quadratus.

De outra parte, o tronco comum resulbante da unizo jo ramus lobi sinistri medlalis e do ramus lobi quadrati, conifigurado naquelas 9 disseceres $(59,4$ - Obs. $1 \mathrm{~m}$, 2f, 7m, 8f, 9m, 11m, i2m, 14m, 15f), encontra-se livre de afluentes em apenas 1 oportunidade $(6,78$ - obs $9 a)$ acolhendo, nas denais preparactes, afluentes de diversas origens, a saber:

a) apenas do lobus quadratus, mais exatamente, cinco coletores isolados, 1 vez ( 6,78 - Obs. $7 \mathrm{~m}$ ), tres afluentes independentes, 1 vez $(6,78$ - Obs. 2f) e um tributario 1 vez (6,78 Obs. 11m);

b) simultaneamente do lobus auadratus, do lobus sinister do lobus caudatus (pars supradortalis), 1 vez ( 6,78 . obs. Im), ou seja, tres coletores isolados do lobus quadratus, seguidas de tronco comum a coletores do lobus sinister do lobus eaudatus (pars supraporta(is);

c) concomitantemente do lobus quadratus e do lobus sinister medialis, 4 vez (6,79. Dbs. 8f), isto tres tributarios isolados do lobus auadratus. seguidas de dois tributarios independentes do lobus sinister medialis, o segundo dos quais consplcuo, entre os quais se posta outro afluente isolado daquela mesma origem;

d) simultaneamente do lobus sinslter lateralis, lobus sinister medialis lobus auadratus, $q$ vez $(6,78$. Obs. 12m), vale dizer, ties contribuiszes deste altimo loba, tronco comum dois afluentes, o primeiro vindo do lobus sinister medialis - o segundo conspieuo, do lobus sinister lateralis. seguida de coletar isolada do lobus quadratus; e) a mesmo tempo do lobus auadratus, do lobus sinister medialis e do lobus caudatus (pars supraportalis), 1 vez $(6,7 \%$ - Obs. $14 \mathrm{~m})$, isto e, dois coletores inoedendentes do bous quadratus entre os quais se posieiona cronee comum dois coletores do lobus sinister medialis, o segundo dos quais conspleuo, dos afluentes do lobus quadratus, o ultimo deles conspleuo, entre os quais se posiciona afluente isolado do lobus caudatus (pars supraportalis);

f) concomitantemente do lobus quadratus e do lobus sinister. Lateralis, 1 vez ( 5,78 - abs. 15f), ou seja, af luente isolado do lobus quadratus, segulda de coletor consplsuo de lobus sinister lateralis.

2) D ramus lobi sinistri lateralls e o ramus lobi sinistri medialis acham-se diretamente conjugados em 5 das 15 preparaczes $(33,09$ - Obs. 3f, $4 f, 6 m$, $10 m, 13 m)$. Amoos mostram-se liveres de tributbrios, 2 vezes $(13,3 \%$ - Obs. $3 f$, $6 \mathrm{~m})$, enquanto que apenas o ramus labj sinistri medialis acolhe eferentes, 1 vez (6,78 - Obs. 4f), vale dizer, tres contingentes isolados do lobus quadratus e, por dltimo, coletor independente conspleuo do lobus sinister mediails.

0 ramus lobi sinistrl lateralls, a seu turno, scolhe eferente isolado do labus quadratus, 1 vez (6,78 - Obs. $13 \mathrm{~m}$ ). Tanto o ranus lobi sinistri lateralis como o ramus lobi sinistri medialis recebem tributarios, 1 vez $(5,6 z$ - Obs. $10 \mathrm{~m})$, isto e, orimeiro deles reolhe dois eferentes isolados do lobus sinister medialis $e$ o segunoo tamoem dois ductos isolaoos do labus quadratus.

o tronco comum resultante da reunizo do pamus lobi sinistri lateralis e do pamus lobi sinistri medialis, observado neste grupo de 5 pecas $(33,0 s$ - Obs. $3 f, 4 f, 5 m$, 10m, 13m), ja representa o proprio ramus principalis sinister, 2 vezes $(13,38$ - Obs. $10 \mathrm{~m}, 13 \mathrm{~m})$ e acolhe tributarios de diversas origens, a saber:

a) simultaneamente do lobus saudatus (pars supraportalis) e do lobus quadratus, 2 vezes $(13,3 \%$ - Obs, $4 f, 1 \mathrm{~m})$, vale dizer, coletor isolado do lobus quadratus, seguido de tributarto conspleuo do lobus eaudatus (pars supraportalis e processus papiliaris) e finalmente mais dois coletores Jaquela mesma origem, 1 vez ( 6,79 - Obs. 4f) tributario independente do lobus quadratus, seguido de coletor conspleuo do lobus eaudatus (pars supraporta(is), 1 vez (5,7\% - Obs. $10 \mathrm{~m})$; 
b) concomitantemente do lobus quadratus, lobus sinister latesalis e loous caudatus (pars supradortalis), ou seja dois coletores independentes do lobus auadratus, tibutario cansoleuo do loous sinister baterabis, mais um af luente consoleur daquela mesma origem e, finalmente, dois afluentes isolados do lobus caudatus (Dars supraportalis), 1 vez (5,7\% - Obs. 3f);

c) do mesmo tempo do lobus sinister medialis $e$ do lobus quadratus, vale dizer, tronco comum associando tres elementos, dois do primeiro looo um do ultimo, 9 vez (5,7\% - Dos. 6m);

d) simultaneamente do Lobus sinister medialis, do lobus caudatus (pars supraportalis) e do lobus quadratus, ou seja, afluente isolado do lobus caudatus (pars supraportalis), tronco comum a dois afluentes, un do lobus sinister medialis e outro do lobus ouadratus e mais un tronco comum associanao quatro componentes, o primeiro oo lobus sinister medialis, conspleuo e os tres ditimos do lobus quadratus, ? - wez (6,78 - Dbs. .13m.

3) O ramus lobi sinistri lateralis e o ramus lobi quadrati achan-se diretamente conjugaoos em 1 dos 15 flgados $(5,7 \%$ - Obs. $5 \mathrm{~m})$, oportunidade em que ambos recolhem contribuictes inominadas, provenientes do lobus sinister nedlatis, ou seja, ao ramus labi quadroti, un of luente isolado a, a ramus loti simistri lateralis, dois tributarios independentes. Quanto ao tronco comum observado na glandula em questso, recebe eferente isolado, conspicuo, do lobus sinister lateralis.

Vejamos agora o modo pelo qual, continuando a confluir, os coletores nominados, integrantes do sistema do ramus principalis sinister, acaban por constitullo.

1) 0 panus lobi sinistri lateralis partieipa da formacso do sistema do ramus principalis sinister, en todos os casos $(100,0 \%)$. Ooservamo-lo associado ao ramus lobj sinistri nedialis, 5 rezes $(33,08$ - Dbs. $3 f, 4 f, 8 m, 10 \mathrm{~m}$, $13 m)$ ao ranus lobi quadrati, 1 vez $(6,75$ - Obs. $5 \mathrm{~m})$. Nas 9 pecas restantes (59,4s - Obs. im, 2f, 7m, 8f, 9m, $11 m, 12 m, 14 m, 15 f), v i m o-10$ a desembocar no tronco comum para o qual convergem, o ramus lobi quadrati e ramus lobi stnistri medialis, sendo visto livpe de contribuiszes em apenas 1 drggo $(6,79$ - Obs. $14 m)$, quando a via resultante, já representativa do proprio ramus principalis sintster vem ter ducto conspicuo do lobus caudatus que reune duas contribuieses da pars supraportalis e uma do processus paDillaris. Nas demais preparactes, acolne contingentes de diversas origens, ou seja:

a) simultaneamente do lobus sinister medialis, lobus audratus e do lobus caudatus (pars supraportalis), 2 vezes $(13,35$ - Obs. $2 f, 7 m)$, isto e, tronco conum de quatro componentes, um do labus auadratus e tres do lobus sinister medialis, o oltimo deles console cuo, eferente inominaoo daquele lobo. seguido de dois contingentes isolados do lobus caudatus (pars supraporta(is), sucedidos por tronco comum de dois componentes, um do lobus quadratus e outro do lobus caudatus (pars supraportal(s) ef inalmente, tributario lsolado, consplecuo desta mesma origem, 1 vez ( $6.7 \%$ - Obs. $2 f)$ e tronco comum de tres elementos oo lobus sinister nedialis, seguido de quatro afluentes alternaoos do labus auadratus e se labus caudatus (pars supraportalis), o oltimo do lobus quadratus, conspleuo - por fim, tronco comum associando dols elementos do lobus caudatus (pars supraportal(s), 1 vez (6,7\% - Obs. $7 \mathrm{mj}$. Nestas 2 preparacobs, 0 ducto assim caracterizado encontra-se live de aftuentes e jt contitui o proprio ramus principalis sinister;

b) concomitantemente do lobus sinister medialis a do lobus caudatus (pars supreportalis), 2 vezes (13,38 - Obs. $12 \mathrm{~m}, 15 \mathrm{f})$, isto $t$, coletor isolado conspleuo do lobus sinister medialis, seguido de dois tributarios do lobus eaudatus (pars supraportalis), o primeiro dos aujis consoleuc que acolhe inclusive contribuiczo do peocessus papillaris, $i$ vez (5,7\% - Obs. 12m) e eferente isolado, consoleuo do loous sinister medialis $e$ outro afluente isolado do lobus caudatus (pars supraportalis) q vez (6,7\% - Obs. 15f). Tambem nestas 2 glandulas, o ducto assim constituldo encontra-se livre de eferentes representa ja o proprio panus principalis sinister;

C) unicamente do lobus sinister medialis, vale dizer, um tributario isolado, vez ( 6,79 - Obs. Im). O ducto comum, aqui caracterizado, encontra-se livre de contribuicoses e representa o proprio ramus prancipalis sinister;

d) concomitantemente do lobus sinister medialis e do loous eavatus bars supraporta(is), ou seja, tronco comum de dois elementos, um de cada una dessas origens, 1 vez $(6,75$ - Obs. (m). O conduto aqui formado representa - ramus princtpalis sinister e acolhe, em seu trajeto, coletores inominacos representados por tronco comum de dois 

capivara (Hydrochoerus hycirochoeris).

componentes, un do lobus sinister medlalis e autro do lobus caudatus (pars supraportalis), seguido de dois afluentes isolados oesta mesma origem;

somente do bobus sinisier medialis, melhor explicanoo, um coletor isolado, 1 vez $(5,7 \%$ - Obs. $11 \mathrm{~m})$. O conduto verificado nesta peca representa o proprio ramus principalis sinister e acolhe, en seu pereurso, ealetor isolado do lobus caudatus hpars supraportalis):

f) ao mesmo tempo do lobus quadratus e lobus caudatus (pars supraportalis) e processus Daplliaris, isto e, um coletor de eada regigo, os dois ditimos en tronco, 1 vez (6,7\% - Obs. Of);

2) D pamus lobi sinistri medialis toma parte na constituica do pamus prineipalis sinister nos 15 deraos estudacos $(100,05)$, ouanda o vimos associaco as ramus lobi auadrati, 9 vezes $(59,4 \mathrm{~g}$. Obs. $1 \mathrm{~m}, 2 \mathrm{f}, 7 \mathrm{~m}$, af, $9 \mathrm{~m}, 11 \mathrm{~m}, 12 \mathrm{~m}$, 14m, 15f) e ao ranus lobi sinistri lateralis, 5 vezes (33,08 - Obs. 3f, 4f, 6m, 10m, 13m). Na peca restante ( 6,68 - Dbs. 5m), surpreendemo-to a desembocar no tronco comum constituida pelo ramus lateralis lobl sinistri pamus lobi quadrati, quando acolhe, en seu trajeto, tres coletores isolados do lobus quadratus. A via aqui formada - la confluencia dos mencionados duecos, caracteriza"o propr 1.0 pamus prineipalis sinister que recebe apenas um af luente do lobus caudatus (pars supraportalist.

3) O ramus lobi quadrati, consoante verificamos, surge como constituinte do sistema do ramus princtpalis sinister en 13 dos 15 figados'estudados (85, 8 \% - Obs. 1m, $2 f, 3 f, 4 f, 5 m, 6 m, 7 m, 8 f$, 9m, 11m, 12m, 14m, 15f). Nas outras 2 pecas $(13,28$ - Obs. 10m, 13m), observamo-lo a enderecar-se ao pamus lobi dextri medialis. No conjunto dessas 13 preparacoroes en que o ramus lobi quadrati faz parte do sistema do ramus prinefpalis sinister, verificar:a-lo formando tronco comum com o pamus lobi sinistri wedialis, 9 vezes $(59,48$ Dbs. 1m, $2 f, 7 m, 8 f, 9 m, 11 m, 12 m$, 14m, 15f) e com o ramus lobi sinistri lateralis, 1 vez $(6,78$ - Obs. $5 \mathrm{~m})$. Nas demais glandulas constituintes deste grupo, vimos o citado ramo a enderecar-se ao bronco comum comoosto zelo ramus lobi sinistri lateralis e ramus lobi sinistri medialls, 3 vezes $(19,8 x$ - Obs. 3f, 4f, 6m), quando se encontra livre de contribuicóes. O ducto comum formado nestas 3 disseceses ja caracteriza o proprio ramus principalis stinister e encontra-se isento de tri- butarias, 2 vezes 613,38 - 00s. 3f. 4f), acolhendo-as na observacgo complementar (5,75 - Obs. 6m), vale dizer, tronco comum de seis comoonentes. tres do lobus sinister laterabis tres do bous cauotius (oars supraoorta(1s), seguido de mais tras afluentes inominados, dois do loous quadratus e - outro do lobus dextri medialis, com plementar ( 6,75 - Dos. Gm), valedizer, tronco comum de seis camponentes, tres do lobus sinister lateralis. tres do lobus caudatus (pars supraportalis), seguido de mais tres afluentes inominados, dois do lobus quadratus - outro do loous dextri medialis, com os dois ditimos compondo tronco comum.

\section{F) TRACTOS ANASTOMOTICDS}

No estudo das 15 preparacjes, evidenciamos, em 2 delas $(13,2)$ - Qbs. $1 \mathrm{~m}$, $5 \mathrm{~m}$ ), a presenca de tracto anastomotico entre diferentes coletores, ou seja:

a) o tronco do ramus lobi sinistri mediais ramus lobi. quadrati (sisteme do pamus prinelpalis sinister) ao pamus lobi dextri medialis (sistema do ramus principalis dexter), 1 vez (6,78. Obs. $1 \mathrm{~m}$ );

b) dois ductos conspicuos vindos do labus caudatus (pars supraportalis), un deles integrante do ramus principalis dexter o outro, do ranus principalis sinfster, 1 vez (6,7s - Obs. 5m);

c) coletor do lobus caudatus (pars supraportalis) ligado a ducto consoleuo de gesma procedencia, e o ramus processi caudati, ambos integrantes do sistema do pamus prineipalis dexter, 1 vez ( 5,78 - Obs. $5 \mathrm{~m})$. O referido ducto conspleuo o mesmo implicado no caso do 1 tem anterior.

\section{COMENTARIOS}

Considerando o fato de os trabathos encontrados sobre o assunto dedicarem-se a outros roedores que nao a cadjuara - e a parte deles, ainda a coethos - o confronto dos resultados desta pesquisa com o conteddo desses trabalinos, far-se-t com certa restricgo, parcialmente atenuada no caso da pesquisa de pRAQR et alii, 6, relativa ao ratso do banhado, ja que o figado desse animal mostra-se looado, como o da especie que estudamos agora os ausares se valeram de igual metodo ao por nos utilizado. Assim sendo, exatamente na $r a t y o$ do banhado que surpreendemos as tres opcôes de formacro do duetus choledocus, como na capivara, ou seja, triconvergeneia do ramus principalis dexter, panus principalis si- 
nister e ductus eysticus $(20,08$ e 66,78), unigo dos dois primeiros coletores (50,08 $20,08)$ associacso do ductus hepaticus com o duetus cysticus $(20,0 \%$ e $13,3 \%)$. As poreentagens indizadas dizem respeito, por oroem, ao ratyo do banhado e a capivara. Para coethos, relatam DIAS et alij, 1 , que a segunda das opcórs citadas a mais freqoentemente encontrada $(94,91 \%)$, pela razo de o ductus cysticus integrar-se entyo ao sistema do ramus principalis dexter, disposicro que aparece no patyo do banhado $(26,7 \%)$ e tambem na capivara $(20,0 \%)$, en menor numero de casos. Esses autores descrevem o coledoco a receber tributarios de varias procedencias, o que ngo registramos em nosso material bem como PRADA et alij. 6, no ratyo do banhado. Alids, o encontro do ductus cysticus assoeiado ao ductus hepaticus surge tanto no ratgo do bantiado $(20,0 \%)$ quanto na capivara $(13,3 \%)$ mas, nyo em coethos. D ductus cysticus integrado 30 sistema 00 ramus principalis sinister, fato que nao observamos em nossas pecas, adarece no $r a t z o$ do gannaoo $(33,38)$ e nos coelnos examinados por DIRS et alii, 1 (5,898), disposicgo que verificamos representada no primeiro dos esquemas que ilustram o trabatho de OLIUEIRA et olii, 4, en cobsios. Ne constituicso do ranus prineipalis dexter, descrevemos aproximadanente a mesma composicyo relatada por PRADR et alij, 5 , com a ressalva de que encontramos raramente (6,75) tamben a participacyo-do ramus lobj quadrati nesse sistema. Para os coelhos, segundo DIRS et alii, 1 , participan desse sistema, mais freqúntemente $(76,478)$, os ramos lateral e medial do lobo direito e ducto elstico. A participacto desses ramos foi bastante freqoente na eapivara mas, a do ductus eysticus foj apenas 19,8\%. De maneira generica, a descricyo de DTTRUIANI: 5, para a cobala parece nao fugir oo que estamos adresentando, Dois esse autor considera como tributbrios do ducto direito, um afluente primbrio do lobo mediano direito, um ou dois do lobo caudato, um af luente primbrio do lobo medial esquerdo bexcepcionalmente) e um lobo quadrado. Em informacyo que parece contraria ao que ora expusemos, OTTRUIANI, $S$, relata qua nzo existem, no coetho, ductos que permitam distinguir dois territorios biliferos, um direito e um esquerdo mas aponta a existencia de un so ducto billfero que nasce por meio de dois afluentes primarios, aos quais chegan outros afluentes primarios e ducto elstico, sendo o ducto coledoco continuacro desse ducto billfero. Na realidade, registramos a ocorrencia freqoente de ser, o pamus principalis dexter, muito eurto e colocarem-se, os seus ramos formadores, em posifzo tal que parece continuar-se, o pamus principalis sinister, diretamente no ductus choledocus. OTTRUIRNI, 5, menciona, mesmo para a cobaia, que o ducto esquerdo é mais longo e fino que o direito. Tambem para a marmota, esse autor 5 refere a constituiggo do ducto billfero intra-heda- tico por varios arluentes dispostos em - $v$. talvez pela mesma razgo que invocamos anteriormente. Esse autor, dor outro lado, alude en varias oportunidades a conspieuo afluente Drimario coletor do lobo eaveato que penetra no colecoso. Emoora nao tennamos relatado o encontro oe tributarios diretos do ductus choledocus na eadivara, entendemos que essa eitacgo de OTTAUIANI, 5 . talvez se prenda ao fato seguinie: assim como o ductus choledocus parece representar direta continuaczo do ramus principalis sinister, em determinados casoso ramus principalis dexter. sempre muito curto, parece representar direta continuaço do ramus processi caudati, pela disposicalo que este assume; como o ramus prinelpalis dexter, nyo apenas na capivara, de modo geral en todos os manlferos, representa um dos formadores do ductus choledocus, pode-se ter a impressyo, mencionada por OTTRUIANI, 5 , de que o coledoco recolne, na sua ultima parte, o alfuente primario do labo eaudato. Para a nutria, indica mesmo que grande quantidade de afluentes bibiferos convergem para tronco que, por sua vez, participa da constituicgo do ducto direito. De fato, registramos, nos 15 casos estudados, o ranus processi caudati a integrar o sistana do ramus principolis dexter, ben como a participaço, nesse sistema, de varibuel numero de coletores inominados vindos nao apenas do processus caudatus, mas tamben do processus papillaris a da pars supraportalis do lobus caudatus, sendo muitos destes uttimos, conspleuos. Para a propria nutria e para a cobaia adulta, DTTAUIANI, 5, refere-se t presenca de um ducto bilifero para cada um dos territorios direito e esquerdo, destacando participacyo do afluente primbrio do lobo medial direjto no ducto oilifero direito - conforme observamos em grande numero oe decas $(92,4 \%)$ - ate excedeionalmente desemcocanoo na grande dilataca formada pelo encontro do ducto direito com o ducto esqueido. Disposicso semelhante registramos $(6,78)$ quando do enderecamento do ramus lobi dextri medialis diretamente na formacgo das vias extra-hepbticas, neste caso como tributario do ductus eysticus. Alias, a participacto do ductus eysticus diretamente na formacto das vias extra-hepaticas representa o aspecto mais comum na composicyo do duetus choledocus da eapivara (56,78). Como o duetus eysticus $(20,08)$, o pamus lobl quadrati $(13,3 \%)$ garticipa raramente do sistema do remus principalis dexter, segundo abservamos na capivara, pois é evidente que se integra com maior freaoencia $(85,8 \%)$, a 5 istema do ramus principalis sinister, estanco mesmo freodentemente unido, o ramus lobi quadrati, diretamente ao ramus lobi sinistri medialis $(59,4 \%)$, embora posicyo topogrbfica do bobo quadrado favoreca sua drenagem, tanto parao sistema do ramus principalis dexter, quanto para o do ramus principalis sinister, encontramos somente pequenos coletores es- 

capivara (Hydrochoenes hydrochoeris).

coanda a direita o ramus lobi quadrati, mais consolcuo, a esquerda, dentro de sua regizo de drenagem e, consegoentemente, tendendo a integrar-se ao segundo dos sistenas. Talvez a zosizac ja vesileulo biliap, entre o lobo quadrado e o looo medial direito, tenta algo a ver com esse fato. Da mesma forma, foram esperados, em nosso material, como mais freqoentes, alguns arranjos encontrados, como a associacto do pamus lobi dextri lateralis e panus processi caudati $(85,85)$, pela proximidade de territorios que escoan.

No tocante ao sistema do ramus principalis sinister, de modo geral a mesma, a composicsa descrita por PRADa et alii, 6, para o ratzo do banhado, da qua registramos, para a capivara, com a ressalva de que ngo indicamos, nessa composifyo, o ductus eysticus e nem contribuicaes inominadas vindas do lobus dexter medialis. Conforme já comentamos, DIAS et alii, 2, relatam. para o coelho, a para participacso do ductus cysticus nesse sistema (5,89\%), disposicro que observamos registrada no primeiro dos esquemas constantes da publicasgo de QLIVEIRR et alii, 4. Tambem nzo vimos em nosso material, coma DTTAUIANI, $\bar{b}$ na nutria, a participasso, sa bem que tida como excepcional, do afluente do lobo medial diceito no ducto esquerdo. o proprio ramus principalis sinister, que registramos sempre, foi caracterizado por QLIVEIRA et alii, 4, para as cobaias, livre de afluentes en 9,0\% das pecas - disposiggo por nos evidenciada paramente $(5,75)$ - a receber, freqoentemente $(91,0 \%)$, tributarios de diversas procedencias - persebemos que, nesta citaço, esses autores referen-se comoasicso do sistema do pamus principalis sinister no qual, camo coletores nominados, ngo fazemos referencta ccmo eles a participacyo do ramus processi papillaris como ducto isolado, embora tenhamos registrado para a capivara, a presinca de coletores do processus papillaris, pois essa regizo restrita e tajs coletores sempre se acham associados a eferentas da pars suprapartalis, alguns dos quais conspicuos. As associasobes mais freqdentes, na composicso do ramus principalis sinister, vimos surgir entre o pamus labi sinistri medialis e a panus lobi quadrati $(59,45)$ ou a ranus lobi sinistr! lateralis $(33,08)$, explicadas pela pasicyo intermediaria da massa glandular drenada pelo primeiro coletor, en relacgo aos outros dois. Tambem se explica facilmente o fato que registramos, como os autores dos trabalhos que estamos citando, de contribuictes do lobus caudatus bars supraportalis e processus dapil(aris) e do lobus quadratus enderecarem-se tanto para a sistema do ramus principalis dexter, quanto para o sistema da ramus principalis sinister, pela razgo de ocuparen a regigo glandular intarmediaria entre os lobos direito e esquerdo. Alias, DIRS et alii, 1 , registram a participacáa do ramo do lobo quadra- do e do ramo do lobo intermedio (talvez corresponda, para nos, à pars supraportalis do (obus caudatus) tanto no sistema do ramo princidal direito quanto esquerdo, encuanto OLIVEIRA et alij, 4, mencionam a presenca do primeiro ramo na comoosiga do sistema do ramus principalis sinister, praticamente metade dos casos $(53,05)$.

Ocorrencia pouco esperada em flgados lobados - comoarativamente ao que acontece en figados nao lobados - e do encontro de vias anastombticas a relacionarem, entre si. diferentes coletores do sistema excretor. Tal fato foi registrado en 13,24, de nossas pecas de eapivara, quando encontramos essa ligaszo estabelecendo-se, de mado geral, enire ductos integrantes do sistema do ramus principalis dexter e do ramus princlpalis sinister. 0 encontro de anastomoses nao el eitado pelos autores referidos. OTTAUIANI, 5 , af irma, mesmo relativamente a cobaia, que nzo se observam anastomoses entre os varios af buentes secundarios aue se unem em leque dara constituir o ducto billfero esquerdo.

\section{CDNCLUSOES}

Do estudo do sistema exeretor. do figado de 15 capivaras (Hydrochoerus hydroehoeris) adultos, machos a femeas, extrajmos as seguintes canclusars:

1) o ductus choledocus, sempre livre de tributarias resulta, mais freadentemente $(56,78)$, da triconvergencia do ramus principalis dexter, ramus principalis sinister ducius cysticus, tambem da unizo direta dos dois primeiros $(19,8 \%)$ ou, ainda do duetus eysticus e ductus heoaticus $(13,38)$;

2) O ductus hepaticus, caracterizado pela confluencia do ramus principalis dexter e ramus principalis sinister, aparece em poucos casos $(13,3 \%)$, livre de triautarios;

3) D ductus eysticus, livre de af buentes $(32,45)$ ou a receber tranco do ramus medialis lobi dextri e ramus labi quadrati $(6,78)$, une-se em triconvergencia ao ramus principalis dexter e ramus principalis sinister para a constituiego do duetus choledocus (66,7\%), incorpora-se ao sistema do ramus principalis dexter $(19,35)$ ou une se ao ductus hepaticus, imoem para a constituisao do duetus eholedocus $(13,38)$;

4) O sistema do ramus principalis dexter acha-se integrado, de modo geral, pelo ramus lobi dextri lateralis $(100,05)$, 
126 MIGLINO. M.A.; PRADA, ILS.; SOUZA. W.M.; D'ERRICO, A.A.

ramus processi caudati (100,0\%), ramus lobi dextri medialis $(92,4 \%)$, ductus eysticus $(19,8 \%)$ e ramus lobi quadrati $(6,7 \%)$, alem de inumeras contpibuicoes inominatas vinotas do lobus dexier lateralis, lobus dexter medialis, lobus caudatus (pars supraportalis, processus papilbaris e processus caudatus) e loous quadratus;

5) o iamus lobi dextri lateralis une-se diretamente ao ramus processi caudati $(85,83), 30$ ramus lobi sextri medialis (6.78) ou vai ter ironco comum aos dois citados coletores $(6,7 \%)$, semore integrado ao sistema do ramus principalis dexter;

6) O ramus processi caudati associa-se diretamente ao ramus lobi dextri lateralis $(85,8 \%)$, ao pamus lobi dextri medialis $(5,7 \%)$ ou conjuga-se a tronco constituldo pelas citadas vias $(E, 7 \%)$, semore incorporaoo ao sistema oo ramus principalis dexter;

7) O ramus labi dextri medialis liga-se diretamente a ramus lobi quadrati $(13,28)$, oo ramus processi caudati $(6,7 \%)$, do ranus labj dextri lateralis $(6,6 \%)$ ou endereca-se a tronco resul. tante da confluencia desses dois d dtimos coletores $(72,7 \%)$. O focado ramo integra o sistema oo ramus principalis dexter $(92,4 \%)$ ou vai ter ao ductus eysticus $(6,78)$;

8) O sistema do ramus prineipalis sinister mostra-se constituldo, de modo geral, Delo ramus lobi sinistri lateralis $(100,0 \%)$, pamus lobi sinistri medialis $(100,0 \%)$ e panus looj ouadrati $(85,8 \%)$, afora contribuicoes inominadas vindas do lobus sinister lateralis, lobus sinister medialis, lobus quadratus e lobus caudatus coars supraportalis e processus papil(aris);

9) O ramus lobi sinistri lateralis une-se diretamente ao pamus labi sinistri medialis $(33,0 \%)$, ao pamus lobi quadrati $(6,7 \%)$ ou vai ter a tronco constituldo pelos dois citados coletores $(59,4 \%)$, sempre incorporado a sistema do ramus principalis sinister;

10) o pamus lobi sinistri medialis as socia-se diretamente ao ramus booj sinistri lateralis $(33,08)$, a ramus lobi quadrati $(59,4 \%)$ ou endereca-se a tronco formado pelas citados coletares $(6,78)$, sempre integrado ao sistema do ramus principalis sinister;
11) O pamus lobi quadrati conjuga-se diretamente ao ramus looi sinistri medialis $(59,4 \%)$, ao ramus lobi sinistri lateralis $(6,7 \%)$, a ramus lobi dextri medialis $(13,38)$ ou vai ter a tronco Dara o qual confluer o ramus lobi sinistri lateralis o o ramus labi stnistri medialis $(19,8 \%)$. D ramo em foco darticipa da formasto do ramus principalis sinister $(85,8 \%$ ) ou do ramus principalis dexter $(13,39)$;

12) vias anastomoticas foran encontradas (13,3\%) intercomunicando diferentes coletores integrantas do sistema co ramus principalis dexter e co ramus princlpalis sinister.

MIGLINO, M.A.; PRADA, I.L.5.; SQUZR, W.M.; D'ER̃RICD, A.A. Contribution to the study of. the liver exeretory system in wild enimals. $y$. Billary ducts of the capyaara CHydrochoerus nydroenoeris). Rev.Fac. Mea.vet. Zootec. Univ. 5 . Poulo, 23 (2):A15-132 1996.

SUMmaRY: The billary duets of 15 caoybaras (Hydrochoerus hydrochoeris), adults ( $10 \mathrm{males}$ and 5 females) Were studied by dissection. The results show that: 1) the ductus choledacus results of the union of ramus principalis dexter, pamus prinelpalis sinister and ductus eysticus (56.78). ramus principolis dexter and ramus peincipablis sinister (19.8s) or ductus hepaticus and ductus cysticus (13.35); 2 ) the ductus hepatlcus is earacterized in 13.38 of the cases and is atuays formed by the convergenee of the panus principalis dexter end ramus principalis sinister; 3 ) the ductus cysticus, has not affluents in some cases ( 92.48$)$; in others case it receives the pamus nedialis looj oextri associated to the ramus bool quadrati (5.7\%). The duetus cystieus cantribues directly to the formation of the auetus choledocus by union with the pamus ppincidalis dexter and the pamus principalis sinister (66.78) op the ductus hepatieus (13.38) and it integrates, sometimes (19.88), the system of the pamus peincloalis dexter systen; 4) the system of the panus principalis dexter is integrated, mainly, by the pamus labi aextrl lateralis $(900.08)$, ramus processi caudati (100.08), pamus lobi dextri medialis (92.45), ductus eysticus (19.88), pamus labi quadrati (5,78), and by inominate llttle ducts from the lobus dexter lataralis, lobus dexter medialls, loous eaudatus (pars supraportalis, processus papillaris ano processus (audatus); 5) the system of the panus principalis sintster is composed, mointy. by the principalis sintster ls composed (qajinty. Oy the lobi oinistri medialis (100.08) end ramus lobi quadrat1. This system is also integrated by inominate little ducts from lobus sinister baterabls, bous sinister meciabis, bobus quadratus
and loous caudatus bars supranetabis ano and loous eaudatus
processus papil (lapis).

UNITERMS: 1. Anatomy of Suldae, 2. Bile ductsi 3. Liveri 4. Uarthrog 
- DIAS, S.M.; SCHALCH, U.A.; SILUA, P.P.; CAMPQS, V.J.M. Consideraç6s sobre as vias billferas de coelho (Dryctolagus cuniculus): Rev.Port. [1.vet., 74:197-202, 1979.

2 - JABLAN-PANTIC, 0 . Caracteristies and comparative rations of intrahepatic bile duets in domestic animals. Acta Vet. Beograd, 13:3-13, 1963.

3 - NICKEL, R.; SCHUMMER, R.; SEIFERLE, E.; SRCK, W.P. The viscera of the domestic mammals. Berlin, Paul Pacey, 1973. 0.115.

4 - oliveIRA, M.C.; ABREU, M.A.F.; SILUA, P.P. Contribution a l'etude des voies biliares chez le cobaye cla- via porcel(us). Systematisation du ramus principalis sinister. Anat. Anz., 146:160-165, 1979.

5 - OTTRUIANI, G. Richerches radiografiche comparative sulle vie bilifere intrahepatiche. Atti ist. veneto sci., 92:1055-1127, 1933.

6 - PRADA, I.L.5.; D'ERRICO, A.A.; BORELLI, $U . ;$ SDGORB SANCHES, F. Contribuifso ao estudo do sistema exeretor do flgado em animais silvestres. III. Vias biliferas do ratro do banhado (Miocastor coypus). (Trabalho aoresentado no i2. Congresso Brasileiro de Rnatomia e 4. Congresso Luso-Brasileiro de Anatomiaj Belo Horizonte, 198i.

Recebido para publicacao em: 20/10/85

Aprovado para publicacjo em: $11 / 12 / 85$

Impresso en $12 / 86$ 


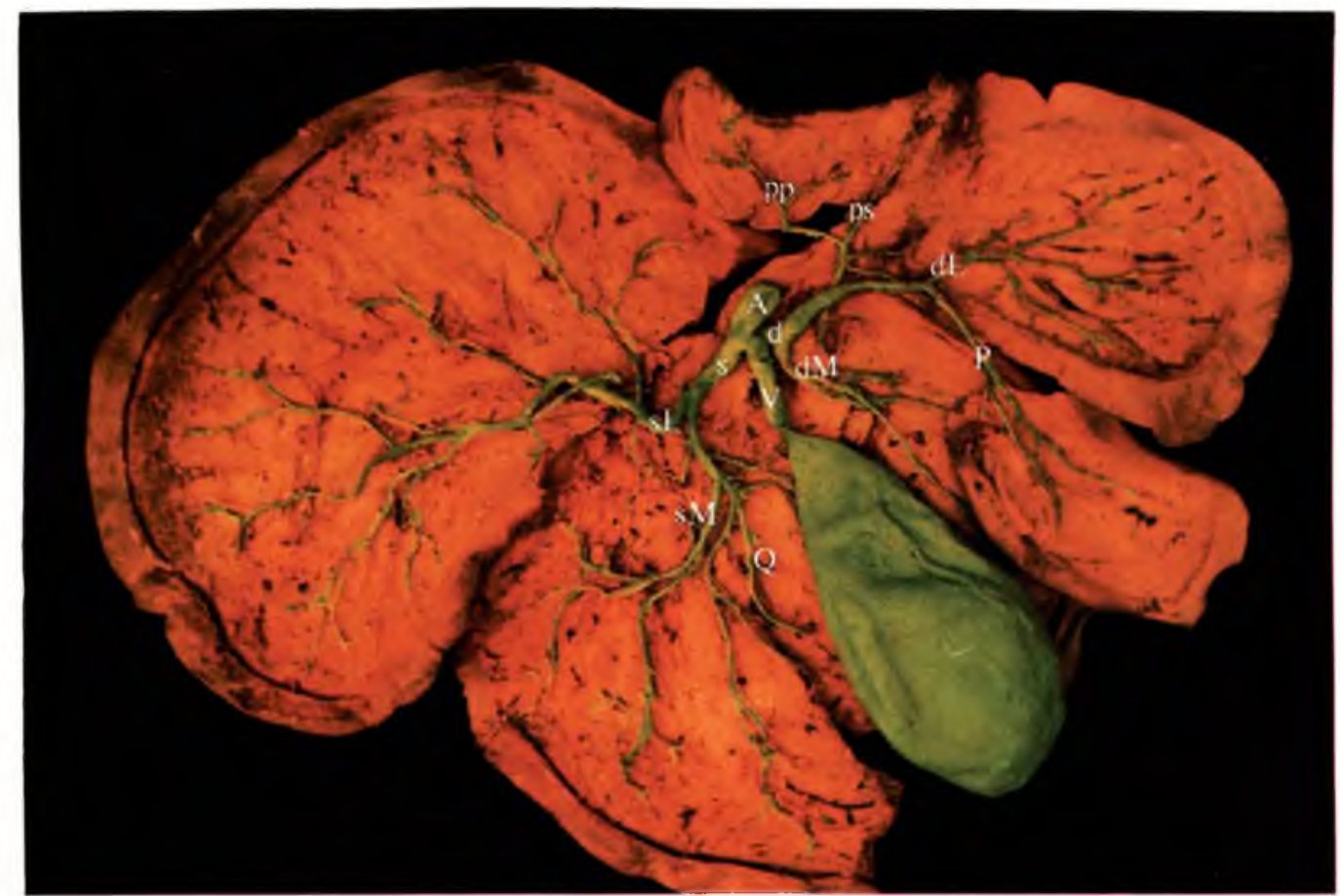

FIGURA 1 - Figado de capivara (Hydrochoerus hydrochoeris) macho, adulto (Obs. 7), visto pela face visceral. Redução de, aproximadamente, 2,5 vezes. O ductus choledocus (A) resulta da convergência do ramus principalis dexter (d) e ramus principalis sinister (s). O primeiro desses ramos acha-se formado pelo ramus lobi dextri lateralis $(\mathrm{dL})$, ramus processi caudati $(\mathrm{P})$, o ramus lobi dextri medialis (dM) e ductus cysticus (V), além de afluentes inominados vindos de diversas regiōes, como os do processus papillaris (pp) e da pars supraportalis ( $\mathrm{ps}$ ) do lobus caudatus. O ramus principalis sinister forma-se pela confluência do ramus lobi sinistri lateralis $(\mathrm{sL})$, ramus lobi sinistri medialis (sM) e o ramus lobi quadrati (Q), afora contribuições de diversas regiơes glandulares. 


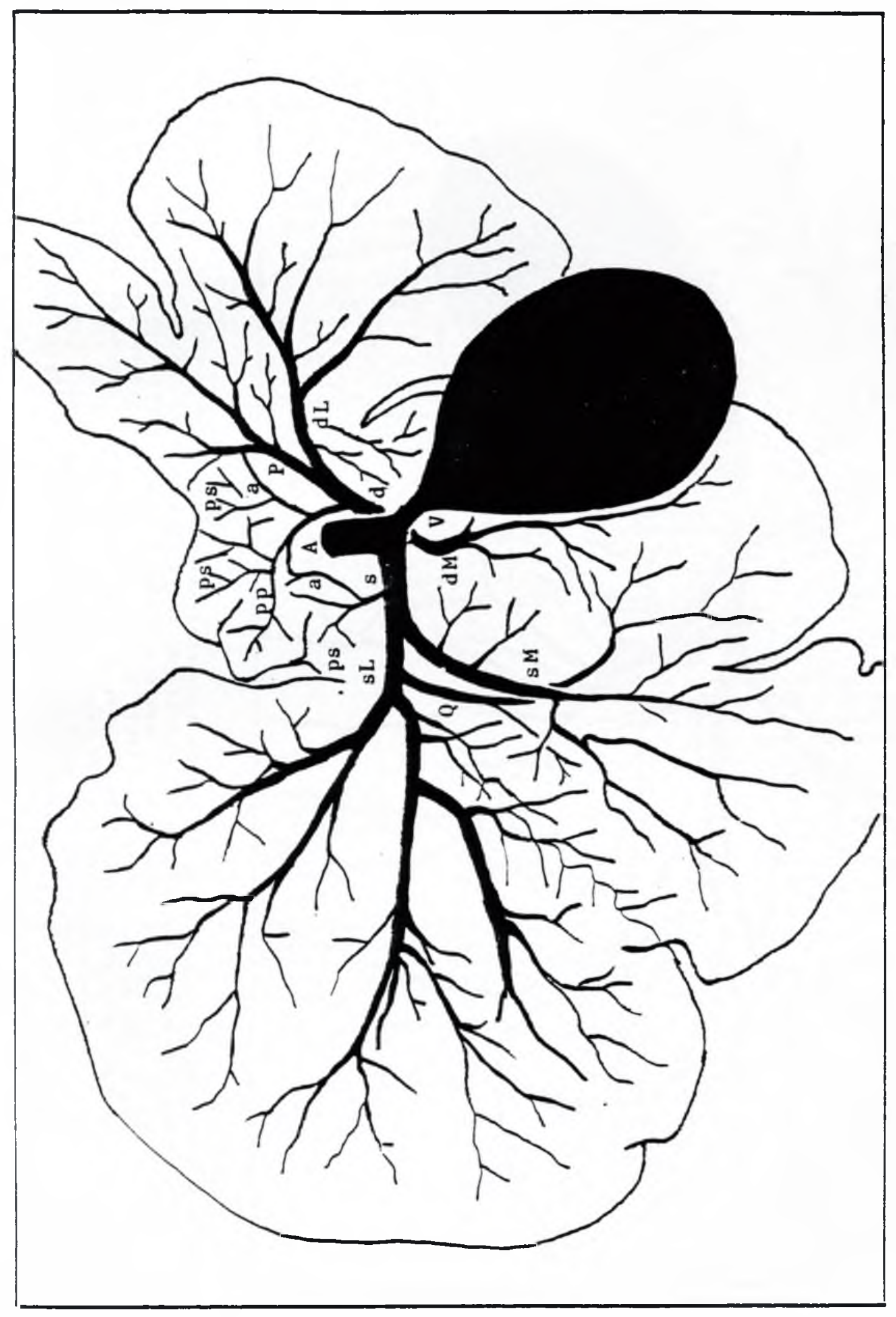

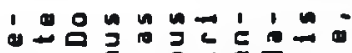

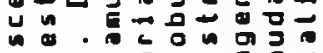

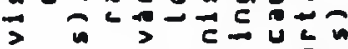
ม 0 음 प บู จ จ - -

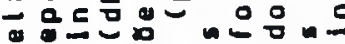

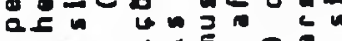
○ ロコニニの゚の。

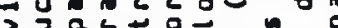

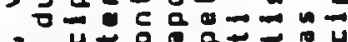

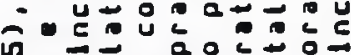
6 는 을움ㄴㄷㄴ은

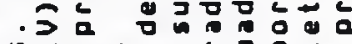

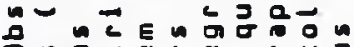
믄

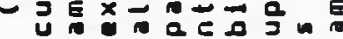
वर्व

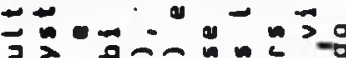

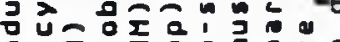

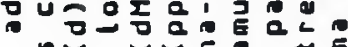

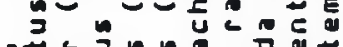

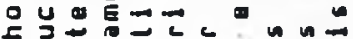

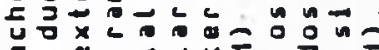

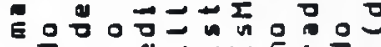
万人

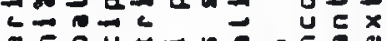

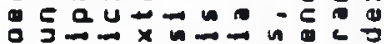

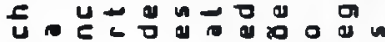

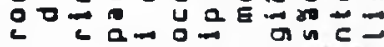

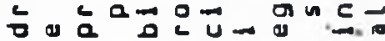

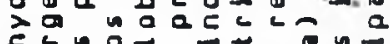

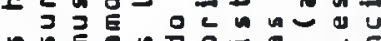
औ 和 它

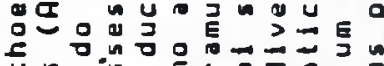
पै ก

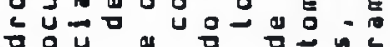

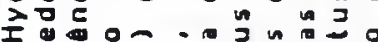

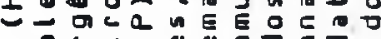
U U $\geq$ 二 >n

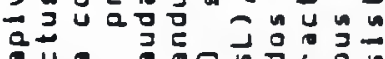
०

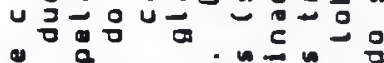
$\therefore$ a ○只

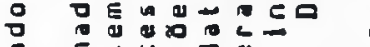

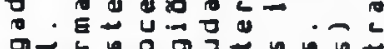

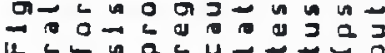
N 


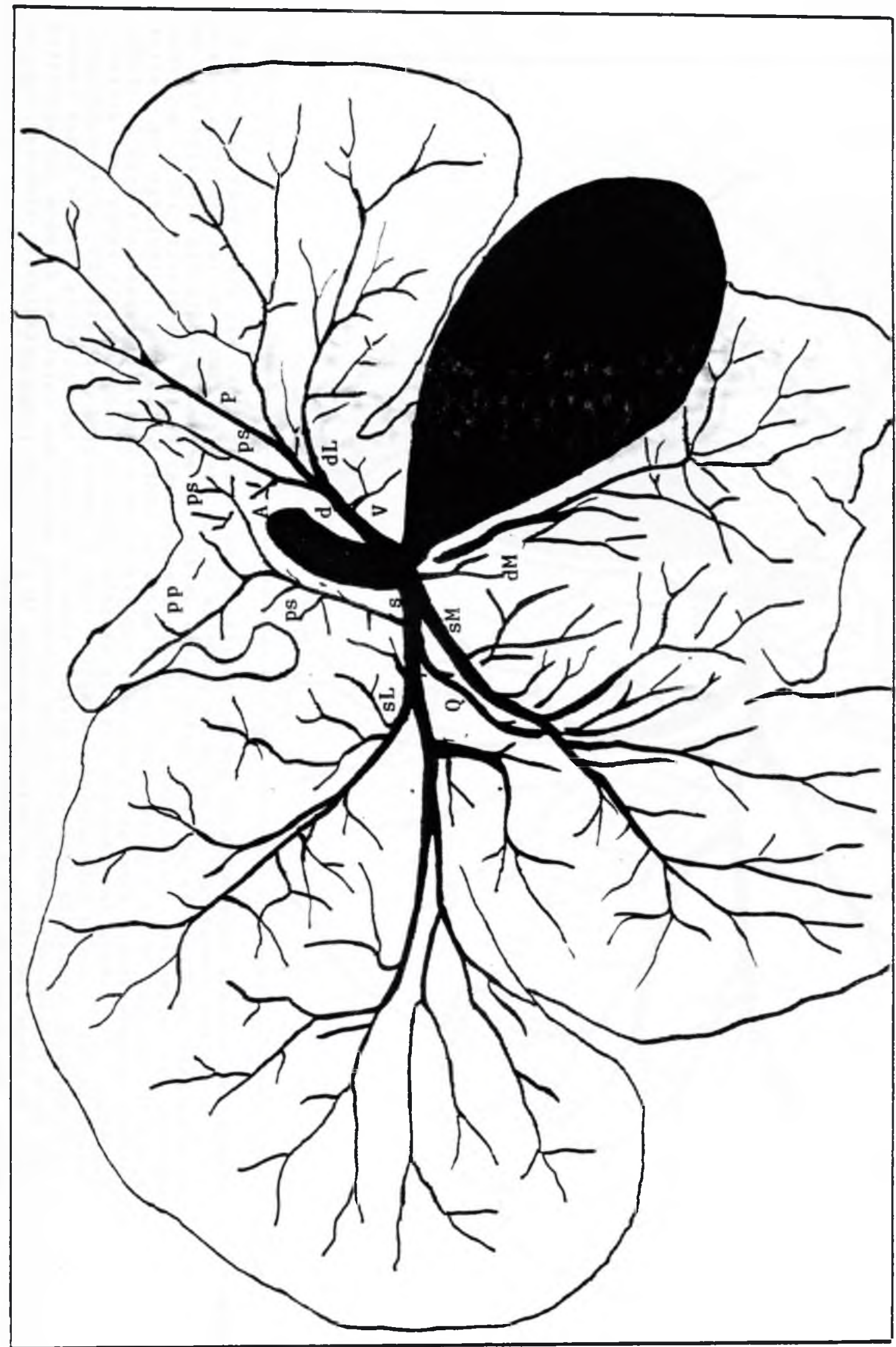

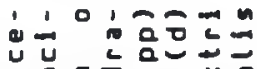

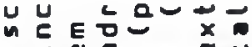

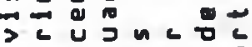
a แั

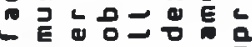

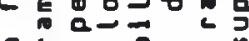
-

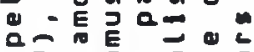

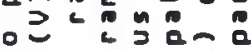

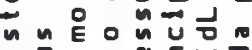
$\rightarrow$ U二 $=0$ - n $\sum_{n}$

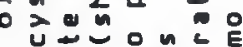

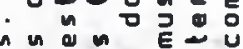

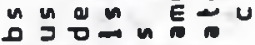

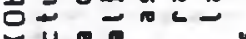

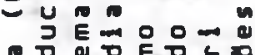

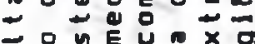
วับ 0

砳药品 更 o.

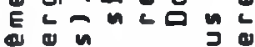

$-\geq-\nabla n$

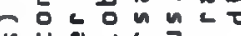
는 皮

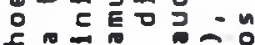

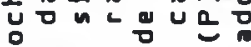
는 >

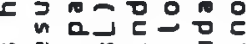
ज \&

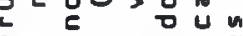

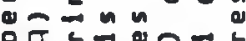

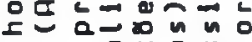

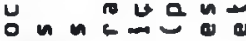
ᄂ >음

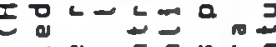

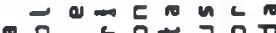
웅

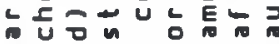

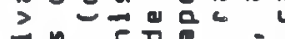
ล

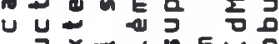
ब $\square 00$ on ㅇ

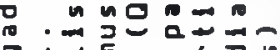

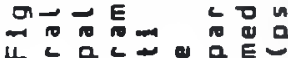

m 